\title{
Comparison of three auto-adjusting positive pressure devices in patients with sleep
} \section{apnoea}

\author{
G.M. Nolan, S. Ryan, T.M. O'Connor and W.T. McNicholas
}

ABSTRACT: Auto-adjustable continuous positive airway pressure (APAP) devices are an emerging treatment alternative to fixed-pressure continuous positive airway pressure (CPAP) therapy for obstructive sleep apnoea syndrome. They have been engineered to automatically adjust the pressure to the optimal level on a continuous basis. However, not all APAP technologies use the same algorithm. Three different APAP devices (Autoset Spirit, Breas PV 10i and RemStar Auto) were compared in a randomised crossover trial in patients already established on fixed-pressure CPAP therapy. The outcome measures were compliance, quality of life and side-effects.

Twenty-seven middle-aged patients (25 male) previously diagnosed with severe obstructive sleep apnoea syndrome (median (interquartile range) apnoea/hypopnoea index 48 (29-76)), established on CPAP therapy for $>3 \mathrm{yrs}$, were randomised to each APAP device for 4 weeks.

Mean pressure and patient compliance were significantly lower on the Breas PV 10i than on the other APAP devices. The devices were similar in terms of quality of life, daytime sleepiness and upper airway side-effects, but patients evaluated them significantly differently in terms of device features, sleep quality and pressure comfort, with the Breas PV 10i being the least popular.

Auto-adjustable positive airway pressure devices differ in pressure delivery and patient compliance in obstructive sleep apnoea syndrome patients.

KEYWORDS: Auto-adjustable positive airway pressure therapy, compliance, continuous positive airway pressure therapy, obstructive sleep apnoea syndrome, quality of life

bstructive sleep apnoea syndrome (OSAS) is a common and potentially serious disorder, affecting $\sim 4 \%$ of adults [1]. It is characterised by repeated episodes of apnoea during sleep, up to several hundred events per night, which lead to hypoxia, sleep fragmentation and excessive daytime sleepiness, with the associated risk of serious sequelae, such as road traffic accidents and significant cardiovascular morbidity and mortality [2-4]. Nasal continuous positive airway pressure (CPAP) is the current therapy of choice, particularly in moderate and severe cases. It has been shown to improve quality of life, reduce the risk of road traffic and occupational accidents, and decrease cardiovascular morbidity and mortality [5-8].

Since the mid-1990s, auto-adjustable positive airway pressure (APAP) devices have been developed to improve patient comfort and compliance. APAP devices are designed to continuously adjust the applied pressure to the optimal level throughout the night, according to an algorithm, and therefore minimise the mean overnight pressure requirements of patients with OSAS $[9,10]$. The effectiveness of APAP in reducing the apnoea/hypopnoea index (AHI), as well as the improvement in symptoms, is similar to that of fixed-pressure CPAP therapy [11-13]. At present, there is insufficient evidence of benefits of APAP devices over traditional CPAP treatment to justify their routine use in clinical practice [14]. The role of APAP in increasing patient compliance, in particular, remains unsolved. Moreover, it is not known whether the different device algorithms have a comparable effect on patient comfort and compliance.

The present study was designed to compare the effect of three APAP devices on compliance, quality of life and side-effects of treatment in patients with OSAS, who were already established on fixed-pressure CPAP therapy.

\section{METHODS}

Subject details

Patients were recruited from the patient population attending the Respiratory Sleep Disorders

\section{AFFILIATIONS}

Respiratory Sleep Disorders Unit, St. Vincent's University Hospital, Dublin Ireland.

\section{CORRESPONDENCE}

W.T. McNicholas

Dept of Respiratory Medicine

St. Vincent's University Hospital

Elm Park

Dublin 4

Ireland

Fax: 35312697949

E-mail:walter.mcnicholas@ucd.ie

Received:

November 012005

Accepted after revision:

March 132006

SUPPORT STATEMENT

The present study was previously published in abstract form: Eur Respir J 2004; 24: Suppl. 48, 566s. 
Unit at St Vincent's University Hospital (Dublin, Ireland). Patients were eligible to participate if they had a confirmed diagnosis of OSAS and were already established on fixedpressure CPAP therapy, using a nasal mask with a device that downloaded time-coded compliance data. Patients diagnosed with a malignant or psychiatric disease, or on regular narcotics, sedatives or psychoactive medications, were excluded. The study protocol was approved by the St Vincent's University Hospital Ethics Committee. Each subject gave written informed consent. Consecutive patients who fulfilled the inclusion criteria were invited to participate.

The baseline characteristics of the subjects are described in table 1 . The patients had been on fixed-pressure therapy for a median (interquartile range) of 53 (37-85) months prior to the start of the study. The following CPAP devices were in use at that time: Sullivan Elite V (ResMed, Abingdon, UK; nine patients), Respironics Aria Lx (Respironics, Inc., Murrysville, PA, USA; 13 patients), RemStar Pro (Respironics, Inc.; four patients), and Fisher \& Paykel HC221 (Fisher \& Paykel Healthcare, Auckland, New Zealand; one patient). Each patient was given a 4-week home trial of three APAP devices: Autoset Spirit (ResMed), Breas PV 10i (Breas Medical, Mölnlycke, Sweden), and RemStar Auto (Respironics, Inc.), set at $4-16 \mathrm{cmH}_{2} 0$, in a randomised crossover trial. The trial was fully blinded to the investigator performing the analysis as this person was not involved in immediate patient contact and was also blinded to the randomisation. It was not possible to fully blind the patients because of the different appearance of the devices. However, patients were not informed about the different technologies used in the APAP devices; they were simply told that these were newer treatment machines. Each patient used the same nasal mask throughout the trial. The devices were assigned to patients in random order by an independent person not involved in the study design, protocol or analysis. At the end of each APAP trial period, patients were evaluated by an experienced sleep technician and physician and asked to complete the Epworth Sleepiness Scale (ESS) [15], a questionnaire about physical and mental health (36-item short-form health survey (SF-36)) [16], and an in-house evaluation questionnaire specifically addressing device features, sleep quality and side-effects (see Appendix). Timecoded compliance data from the fixed-pressure CPAP device at the start of the trial and from the APAP devices at the end of each 4-week trial were downloaded. At the end of the study, patients were asked to place the four devices (three APAP plus $\mathrm{CPAP}$ ) in order of preference and to give reasons for their first preference.

\section{APAP technologies}

The three devices all use flow signal analysis as the basis for their algorithms, but in a different manner to each other. The Autoset Spirit reviews the shape of the inspiratory flow curve on a breath-by-breath basis. A normal unobstructed breath gives a smooth rounded curve shape, but, as the upper airway narrows, flattening of the curve occurs, altering the shape. The degree of flattening determines the response of the device.

The Breas PV 10i creates a model of the patient's breathing signal, which is then compared several times per second to a grid containing 64 pre-stored templates of normal and abnormal respiratory events. The template closest to the patient model determines the response of this unit.

The RemStar Auto compares the inspiratory flow shape of each breath to a rolling patient database, derived from the previous 2-3 min of breath shapes. Two pressure tests are then performed, one which reduces the pressure until the airway begins to collapse and another which increases the pressure until there is no further improvement in the flow shape. A therapy mode is then entered, providing a constant ideal pressure until the next change in respiration occurs.

All three devices also have a safety feature, which increases pressure rapidly in the presence of an unexpected abnormal event.

\section{Statistical analysis}

Subject baseline characteristics and downloaded device parameters are presented as median (interquartile range) or mean $\pm \mathrm{SD}$, depending on distribution, and comparisons were made using a paired t-test or the Wilcoxon signed-rank test for paired samples. A significance level of $\mathrm{p}<0.05$ was considered significant.

\section{RESULTS}

\section{Subjects}

Twenty-seven consecutive patients who met the inclusion criteria were recruited. No subject refused participation and none were lost during the study. Patients were predominantly male (two females) and obese and had previously been diagnosed with severe OSAS disease, as indicated by the median (interquartile range) AHI of 48 (29-76) and ESS score of 15 (9-19; table 1) and compatible clinical signs.

\section{Compliance and treatment pressure}

After each 4-week treatment period, the devices were downloaded for evaluation of compliance and applied pressure. The mean applied pressure was significantly lower on all three APAP devices compared to CPAP, with the lowest mean pressure of $5.8 \mathrm{cmH}_{2} \mathrm{O}$ recorded on the Breas PV 10i. Overall, compliance was acceptable (used for $>70 \%$ nights and $>4 \mathrm{~h} \cdot$ night $^{-1}$ ) on CPAP and on the RemStar Auto and the Autoset Spirit, but was significantly lower on the Breas PV 10i (table 2).

\section{Quality of life and subjective sleepiness}

Analysis of the SF-36 quality-of-life questionnaire showed no significant differences between the three APAP devices or between the APAP devices and those values obtained using fixed-pressure CPAP therapy in all domains. Subjective sleepiness was evaluated using the ESS. Initially, the ESS score was reduced by fixed-pressure CPAP therapy from a pretreatment diagnostic baseline of $15(9-19)$ to $5(3-11)(p=0.002)$, but there was no further significant change after treatment with any of the three APAP devices.

\section{Patient evaluation of the three APAP devices}

All subjects evaluated the devices after each treatment period in terms of device features, sleep quality and side-effects (figs. 1-3). Overall, all three APAP devices were equally easy to use. The Autoset Spirit was the least preferable machine in terms of size and noise, whereas the RemStar Auto represented 
TABLE 1 Baseline characteristics

$\begin{array}{lc}\text { Subjects } & 27 \\ \text { Males } & 25 \\ \text { Age } \mathbf{~ y r s ~} & 53(48-67) \\ \mathbf{B M I} \mathbf{~ k g} \cdot \mathbf{m}^{-\mathbf{2}} & 36.2(31.3-38.6) \\ \text { Diagnostic AHI events } \cdot \mathbf{h}^{-1} & 48(29-76) \\ \text { Diagnostic ESS score } & 15(9-19) \\ \text { Mean fixed-pressure CPAP } \mathbf{~} \mathbf{m}_{\mathbf{2}} \mathbf{O} & 10(8-12) \\ \text { Mean prior CPAP treatment time } \mathbf{m o n t h s} & 53(37-85)\end{array}$

Data are presented as $\mathrm{n}$ or median (interquartile range). BMI: body mass index; AHI: apnoea/hypopnoea index; ESS: Epworth Sleepiness Scale; CPAP continuous positive airway pressure.

the best device features in the evaluation (fig. 1). The Breas PV $10 \mathrm{i}$ provided significantly poorer sleep quality in comparison to the other two APAP devices, which were similar in this respect (fig. 2). All three devices were similar in causing nasal and upper airways side-effects. Pressure discomfort was reported most frequently using the Breas PV 10i (fig. 3).

\section{Patient preference}

Fourteen (52\%) patients preferred an auto-adjusting device over the fixed-pressure device at the end of the trial, with 13 $(48 \%)$ choosing to remain on the original CPAP device. Six patients who preferred the APAP devices chose the RemStar Auto, five the Autoset Spirit and three the Breas PV 10i. For $56 \%$ of patients, "ease of use" was the main reason for selecting the machine of choice, with $22 \%$ listing "noise of device" as the second reason. No patient selected "value for money" as a reason for end preference.

\section{DISCUSSION}

APAP devices are an emerging treatment alternative to fixedpressure CPAP therapy. Several randomised studies have shown that APAP therapy is effective at lowering the AHI and improving subjective sleepiness [11-13]. However, only limited data are available comparing different APAP devices in terms of objective and subjective effectiveness. Various authors have suggested that the efficacy of different APAP devices in improving objective sleep parameters varies [17-19]. In a randomised crossover trial of $12 \mathrm{CPAP}$-naive patients, three different APAP devices and fixed-pressure CPAP were used, each for 1 night. All three APAP devices produced a significant reduction in the AHI, but there was considerable difference in the efficacy of the devices, as only two of the APAP devices reduced the AHI to the optimal treatment level of $<5$ events $\cdot h^{-1}$ [17]. Similarly, PEVERnAGIE et al. [18] compared the AutoSet device (ResMed) and the SOMNOsmart system (Weinmann Diagnostics, Hamburg, Germany) in a split-night protocol and detected small, but significant, differences in polysomnographic parameters in favour of the AutoSet. However, this study compared a titration device with a treatment device and, therefore, the different settings might have contributed to the variation in results obtained.

The present study is the first report comparing three different APAP devices in terms of delivered pressure profile, patient compliance, quality of life and side-effects in patients already established on fixed-pressure CPAP therapy. Although all three of the APAP devices studied use the flow signal to determine the required pressure, the different algorithms account for a significant difference in delivered pressure, and, consequently, for differences in subjective outcome parameters and compliance. No previous study has compared these same three devices. However, in comparing different APAP devices, similar observations of efficacy and pressure profile have been obtained. FARRE et al. [20] assessed five different APAP devices in a bench study utilising a breathing waveform generator and concluded that the responses of the devices to apnoeas, hypopnoeas, flow limitation and snoring were considerably different. HERTEGONNE et al. [21] came to a similar conclusion in a patient population. They compared the comfort parameters and pressure profiles of the AutoSet and SOMNOsmart in a split-night crossover protocol and detected significant differences.

Only one other trial to date has compared different APAP technologies in a patient group over a longer treatment period. SENN et al. [22] evaluated 29 CPAP-naive patients with OSAS and compared fixed-pressure CPAP and two APAP technologies, each for 1 month, in a crossover design. This study did not find any significant differences between the two APAP devices in terms of symptoms, AHI, mean applied pressure or compliance. At the end of the trial, $72 \%$ of patients had no preference for either APAP or CPAP, with four (14\%) choosing APAP and four CPAP. These results differed from the present study, in which 52\% preferred APAP and 48\% CPAP. However, similar to the present results, they too found that details such as size, weight and noise level during use were the most frequently cited reasons for final preference. Furthermore, the present study evaluated a group of patients already established on fixed CPAP. Such patients were

TABLE 2 Compliance and treatment pressure

\begin{tabular}{|c|c|c|c|c|}
\hline Nights used \% & $100(94-100)$ & $100(79-100)$ & $96(42-100)$ & $59(17-93)^{\star \star}$ \\
\hline Mean pressure $\mathrm{cmH}_{2} \mathrm{O}$ & $10(8-12)$ & $7.3(6.0-9.1)^{\star . \#}$ & $8.0(7.2-10.4)^{\#}$ & $5.3(4.5-6.8)^{\star *}$ \\
\hline Maximum pressure $\mathrm{cmH}_{2} \mathrm{O}$ & $10(8-12)$ & $13.4(9.4-16.0)^{\#}$ & $12.2(10.2-13.4)$ & $10.2(7.2-12.4)$ \\
\hline
\end{tabular}

Data are presented as median (interquartile range). CPAP: continuous positive airway pressure. *: $p<0.05$ versus AutoSet Spirit; **: $p<0.01$ versus CPAP, RemStar Auto and Autoset Spirit; ${ }^{\#}: \mathrm{p}<0.01$ versus CPAP. 


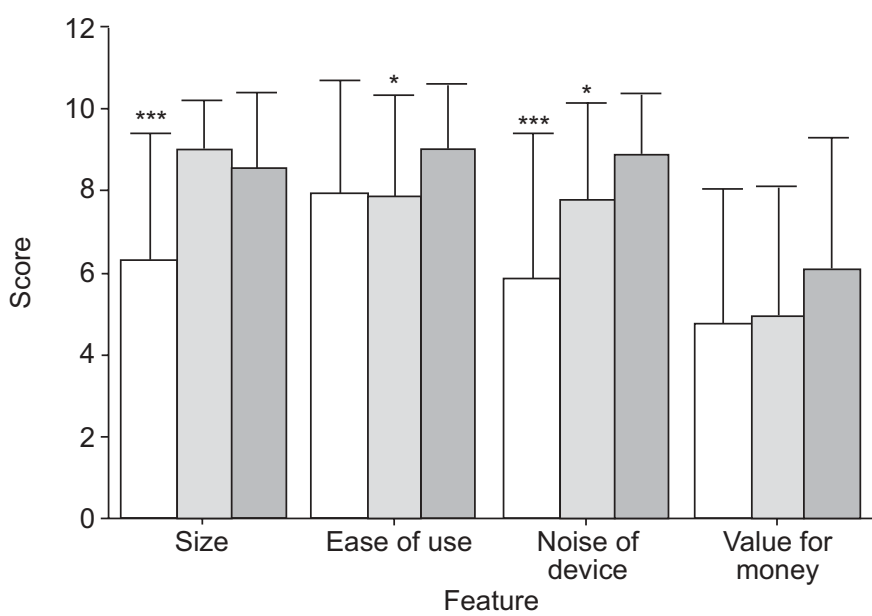

FIGURE 1. Subjective evaluation of the device features of three autoadjustable positive airway pressure devices. The devices were graded on a scale of 1-10, with a score of 1 indicating a strongly negative response and 10 a strongly positive response. $\square$ : Autoset Spirit; $\square$ : Breas PV 10i; $\square$ : RemStar Auto. Data are presented as mean \pm SD. ${ }^{*}: p<0.05$ versus RemStar Auto; ${ }^{* *}: p<0.001$ versus Breas Pv $10 \mathrm{i}$ and RemStar Auto.

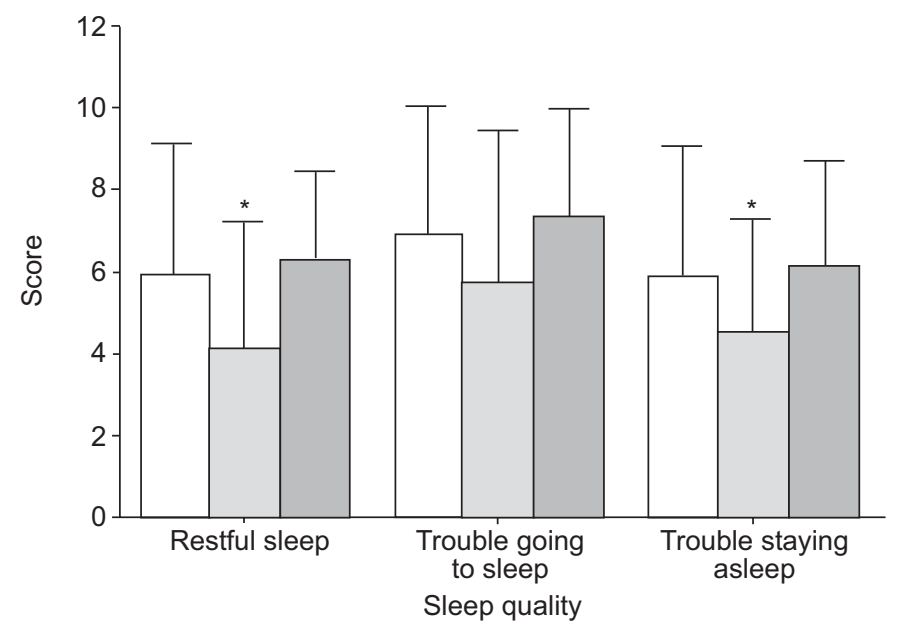

FIGURE 2. Subjective evaluation of sleep quality using three auto-adjustable positive airway pressure devices. The devices were graded on a scale of 1-10, with a score of 1 indicating a strongly negative response and 10 a strongly positive response. $\square$ : Autoset Spirit; $\square$ : Breas PV 10i; $\square$ : RemStar Auto. Data are presented as mean \pm SD. *: $p<0.05$ versus Autoset Spirit and RemStar Auto.

selected because they are familiar and compliant with this type of treatment process, and local airway pathology, such as oedema and inflammation, is likely to have already subsided with CPAP therapy. Also, these patients are established on their mask system, and could, therefore, more accurately evaluate the different APAP technologies without the inevitable limitations associated with CPAP-naive patients.

However, it is recognised that, since the patients were already established on CPAP, there may have been a bias towards CPAP over APAP in the present study. Some previous reports have indicated a preference towards APAP in treatment-naive patients $[23,24]$, whereas others have reported no such

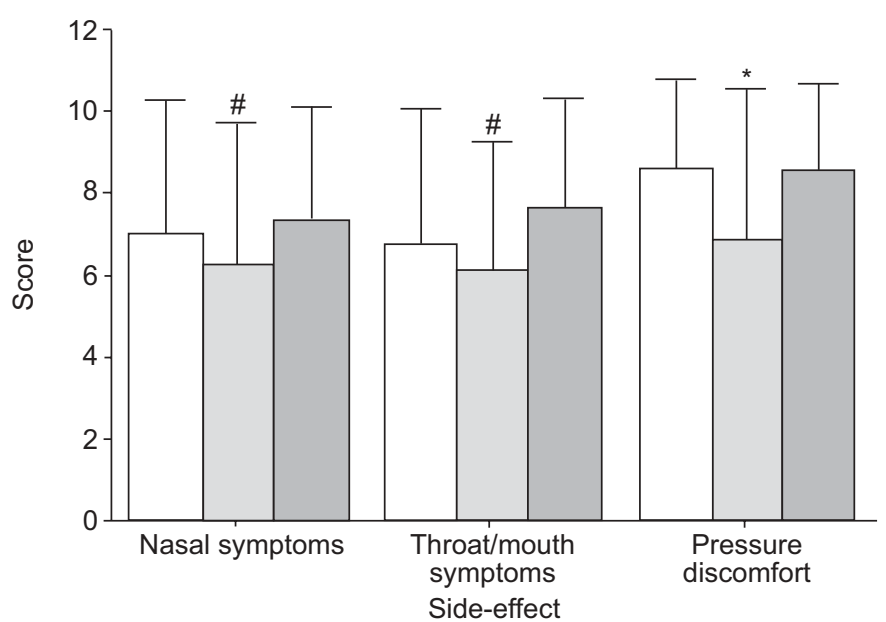

FIGURE 3. Subjective evaluation of treatment side-effects of three autoadjustable positive airway pressure devices. The devices were graded on a scale of $1-10$, with a score of 1 indicating a strongly negative response and 10 a strongly positive response. $\square$ : Autoset Spirit; $\square$ : Breas PV 10i; $\square$ : RemStar Auto. Data are presented as mean \pm SD. *: $p<0.05$ versus Autoset Spirit and RemStar Auto; \# : $\mathrm{p}<0.05$ versus RemStar Auto.

preference [25, 26]. However, the present study design was specifically chosen to directly compare these three APAP devices, an objective that would have been more difficult in treatment-naive patients. There are only limited data on APAP therapy in patients with mild OSAS, although a preliminary report from the current authors' Respiratory Sleep Disorders Unit indicates no consistent preference for APAP over CPAP therapy in patients with mild disease [27].

Another potential limitation of the present study is the inclusion of only two female subjects. This distribution does not allow a conclusion regarding sex differences. However, these sex frequencies represent the distribution of severe OSAS in the current authors' Respiratory Sleep Disorders Unit, and separate studies investigating APAP evaluation in females should be performed specifically targeting this question.

It was found that the Breas PV 10i resulted in a significant lower pressure profile, but poorer sleep quality and more complaints of pressure discomfort; the lowest compliance rates were also observed with this device. Furthermore, it was the least popular choice among patients preferring APAP devices at the end of the study. Unfortunately, an evaluation of the AHI data is not available on this machine, making it impossible to propose ineffective treatment response as a cause of its negative impact. Both the RemStar Auto and the Autoset Spirit reduced the AHI to $<5$ events $\cdot h^{-1}$ (data not shown), but, given the significantly lower pressure with the Breas PV 10i, the same efficacy may not have been achieved. This could have led to impairment of sleep quality, with subsequent lower compliance.

Interestingly, none of the APAP devices led to a change in quality of life, as assessed by the SF-36, or daytime sleepiness, indicating that APAP treatment does not provide additional benefits in these domains. This is of clinical importance as it indicates that patients already established on fixed-pressure 
CPAP therapy will not obtain further benefit by switching to an APAP device.

It is concluded from the present findings that the different APAP algorithms create pressure profiles that are not equally effective in terms of compliance, sleep quality and side-effects in patients established on CPAP therapy. A routine switching from fixed-pressure $\mathrm{CPAP}$ to APAP treatment cannot be recommended, but, if a patient wishes to try APAP treatment, the specific device needs to be carefully assessed in order to ensure treatment effectiveness.

Furthermore, the data do not support the routine prescription of auto-adjustable positive airway pressure as initial therapy for severe obstructive sleep apnoea syndrome, and the present authors recommend that, at present, auto-adjustable positive airway pressure therapy be reserved for patients having difficulty with standard continuous positive airway pressure, or selected patients for whom there is objective evidence of auto-adjustable positive airway pressure superiority, such as those requiring high fixed-pressure levels with continuous positive airway pressure [28].

\section{APPENDIX: IN-HOUSE EVALUATION QUESTIONNAIRE EVALUATION REPORT}

NAME:

DATE:

HOSP. NO:

DEVICE:

The answers to these questions are graded on a scale of 1-10 where 1 indicates a strongly negative response and 10 a strongly positive response.

Please complete this questionnaire by circling the number between 1 and 10 that best reflects your opinion.

\section{A. WHAT DO YOU THINK ABOUT THE SIZE OF THE DEVICE?}

12345678910

$(1=$ too bulky $)(10=$ perfect size $)$

\section{B. DO YOU FIND THE DEVICE EASY TO USE?}

12345678910

$(1=$ too difficult $)(10=$ very easy $)$

\section{IS THE DEVICE VERY NOISY?}

12345678910

$(1=$ too loud $)(10=$ very quiet $)$

\section{WHAT ABOUT THE COST OF THE DEVICE?}

12345678910

$(1=$ too expensive $)(10=$ excellent value $)$

E. WHAT IS YOUR OVERALL IMPRESSION OF THE DEVICE?

12345678910

$(1=$ not impressed at all $)(10=$ very impressed $)$

\section{F. HOW RESTFUL WAS YOUR SLEEP?}

12345678910

$(1=$ not restful at all $)(10=$ very restful $)$

\section{G. HOW MUCH TROUBLE DID YOU HAVE GETTING TO} SLEEP?

12345678910

$(1=$ a lot of trouble $)(10=$ no trouble at all $)$

H. DID YOU HAVE TROUBLE STAYING ASLEEP DURING THE NIGHT?

12345678910

$(1=$ a lot of trouble $)(10=$ no trouble at all $)$

I. ARE YOU HAVING PROBLEMS WITH A BLOCKED, RUNNY OR STINGING NOSE?

12345678910

$(1=$ a lot of problems $)(10=$ no problems at all $)$

J. DID YOU HAVE A SORE OR DRY MOUTH OR THROAT?

12345678910

$(1=$ very much so $)(10=$ not at all $)$

\section{K. HOW MUCH DISCOMFORT DID YOU GET FROM THE PRESSURE?}

12345678910

$(1=$ a lot of discomfort $)(10=$ none at all $)$

The authors would like to thank Resmed (Abingdon, UK), Breas Medical (Mölnlycke, Sweden) and Respironics, Inc. (Murrysville, PA, USA) for kindly supplying the devices and all of the patients for participating in this study.

\section{REFERENCES}

1 Young T, Palta M, Dempsey J, Skatrud J, Weber S, Badr S. The occurrence of sleep-disordered breathing among middle-aged adults. N Engl J Med 1993; 328: 1230-1235.

2 Young T, Blustein J, Finn L, Palta M. Sleep-disordered breathing and vehicle accidents in a population-based sample of employed adults. Sleep 1997; 20: 608-613.

3 Peppard PE, Young T, Palta M, Skatrud J. Prospective study of the association between sleep-disordered breathing and hypertension. N Engl J Med 2000; 342: 1378-1384.

4 Shahar E, Whitney CW, Redline S, et al. Sleep-disordered breathing and cardiovascular disease: cross-sectional results of the Sleep Heart Health Study. Am J Respir Crit Care Med 2001; 163: 19-25.

5 Jenkinson C, Davies RJ, Mullins R, Stradling JR. Comparison of therapeutic and subtherapeutic nasal continuous positive airway pressure for obstructive sleep apnea: a randomized prospective parallel trial. Lancet 1999; 353: 2100-2105. 
6 Montserrat JM, Ferrer M, Hernandez L, et al. Effectiveness of CPAP treatment in daytime function in sleep apnea syndrome: a randomized controlled study with an optimized placebo. Am J Respir Crit Care Med 2001; 164: 608-613.

7 Doherty LS, Kiely JL, Swan V, McNicholas WT. Long-term effects of nasal CPAP therapy on cardiovascular outcomes in the sleep apnea syndrome. Chest 2005; 127: 2076-2084.

8 Marin JM, Carrizo SJ, Vicente E, Agusti AG. Long-term cardiovascular outcomes in men with obstructive sleep apnoea-hypopnoea with or without treatment with continuous positive airway pressure: an observational study. Lancet 2005; 365: 1046-1053.

9 Berthon-Jones M, Lawrence S, Sullivan CE, Grunstein R. Nasal continuous positive airway pressure treatment: current realities and future. Sleep 1996; 19: Suppl. 9, S131-S135.

10 Teschler H, Berthon-Jones M. Intelligent CPAP systems: clinical experience. Thorax 1998; 53: Suppl. 3, S49-S54.

11 Noseda A, Kempenaers C, Kerkhofs M, Braun S, Linkowski P, Jann E. Constant vs auto-continuous positive airway pressure in patients with sleep apnea hypopnea syndrome and a high variability in pressure requirement. Chest 2004; 126: 31-37.

12 Hukins C. Comparative study of autotitrating and fixedpressure CPAP in the home: a randomized, single-blind crossover trial. Sleep 2004; 27: 1512-1517.

13 Randerath WJ, Schraeder O, Galetke W, Feldmeyer F, Ruhle KH. Autoadjusting CPAP therapy based on impedance efficacy, compliance and acceptance. Am J Respir Crit Care Med 2001; 163: 652-657.

14 Berry RB, Parish JM, Hartse KM. The use of auto-titrating continuous positive airway pressure for treatment of adult obstructive sleep apnea. An American Academy of Sleep Medicine review. Sleep 2002; 25: 148-173.

15 Johns MW. A new method for measuring daytime sleepiness: the Epworth Sleepiness Scale. Sleep 1991; 14: 540-545.

16 Ware JE, Snow KK, Kosinski M, Gandek B. SF-36 Health Survey Manual and Interpretation Guide. Boston, MA, New England Medical Center, The Health Institute, 1993.

17 Stammnitz A, Jerrentrup A, Penzel T, Peter JH, Vogelmeier C, Becker HF. Automatic CPAP titration with different self-setting devices in patients with obstructive sleep apnoea. Eur Respir J 2004; 24: 273-278.
18 Pevernagie DA, Proot PM, Hertegonne KB, Neyens MC, Hoornaert KP, Pauwels RA. Efficacy of flow- vs impedance-guided autoadjustable continuous positive airway pressure: a randomized cross-over trial. Chest 2004; 126: 25-30.

19 Shi HB, Cheng L, Nakayama M, et al. Effective comparison of two auto-CPAP devices for treatment of obstructive sleep apnea based on polysomnographic evaluation. Auris Nasus Larynx 2005; 32: 237-241.

20 Farre R, Montserrat JM, Rigau J, Trepat X, Pinto P, Navajas D. Response of automatic continuous positive airway pressure devices to different sleep breathing patterns: a bench study. Am J Respir Crit Care Med 2002; 166: 469-473.

21 Hertegonne KB, Proot PM, Pauwels RA, Pevernagie DA. Comfort and pressure profiles of two auto-adjustable positive airway pressure devices: a technical report. Respir Med 2003; 97: 903-908.

22 Senn O, Brack T, Matthews F, Russi EW, Bloch KE. Randomized short-term trial of two autoCPAP devices versus fixed continuous positive airway pressure for the treatment of sleep apnea. Am J Respir Crit Care Med 2003; 168: 1506-1511.

23 Juhasz J, Becker H, Cassel W, Rostig S, Peter JH. Proportional positive airway pressure: a new concept to treat obstructive sleep apnoea. Eur Respir J 2001; 17: 467-473.

24 d'Ortho MP, Grillier-Lanoir V, Levy P, et al. Constant vs. automatic continuous positive airway pressure therapy: home evaluation. Chest 2000; 118: 1010-1017.

25 Sharma S, Wali S, Pouliot Z, Peters M, Neufeld H, Kryger M. Treatment of obstructive sleep apnea with a self-titrating continuous positive airway pressure (CPAP) system. Sleep 1996; 19: 497-501.

26 Ficker JH, Wiest GH, Lehnert G, Wiest B, Hahn EG. Evaluation of an auto-CPAP device for treatment of obstructive sleep apnoea. Thorax 1998; 53: 643-648.

27 Nolan GM, Doherty LS, Goodman PG, McNicholas WT. Comparison of auto-adjusting and fixed positive airway pressure therapy in patients with mild to moderate obstructive sleep apnoea syndrome (OSAS). Eur Respir J 2003; 22: Suppl. 45, 95s.

28 Massie CA, McArdle N, Hart RW, et al. Comparison between automatic and fixed positive airway pressure therapy in the home. Am J Respir Crit Care Med 2003; 167: 20-23. 